\title{
Real-time Voltage Stability Monitoring in Smart Distribution Grids
}

\author{
Khaled Alzaareer \& Maarouf Saad \\ Department of Electrical Engineering \\ Ecole de Technologie Superieure \\ Montreal, Quebec, Canada \\ e-mail: kaledz87@yahoo.com
}

\begin{abstract}
As electric distribution grids continue in hosting high penetration levels of renewable Distributed Generation (DG), several challenges will be brought to system operation and stability. When distribution networks face either an outage (or unavailability) of DG unit or an increasing in load demand, the loss of voltage stability may result. Moreover, some types of DGs, especially fixed speed induction generators, can cause voltage instability since they always consume reactive power. This paper uses a nodal model for voltage stability monitoring, which is applicable for online voltage control in distribution networks. The load impedance can be easily calculated and the entire distribution system can be simplified as an equivalent impedance based on SCADA and PMUs data. Since the proposed method is based on nodal method, the equivalent impedance will reflect the nonlinear dynamic nature of the load of multi bus power system. An index based on impedance matching theorem will be derived to calculate the voltage stability margin and determine the weak buses, and enable a warning in case of voltage instability detection. The proposed voltage stability monitoring will be performed on a 77-bus, 11 $\mathrm{kV}$ radial distribution system under different operational conditions.
\end{abstract}

Keywords- Voltage Stability Monitoring; Distribution Networks; Thevenin Equivalent Circuits; Distributed Generation; Phasor Measurement Units; Nodal Model.

\section{INTRODUCTION}

Renewable distributed generation has been widely integrated into power systems in the last decades. In spite of its advantages in power loss minimization and voltage profile improvement, integration high penetration levels of DG will pose more challenges in voltage stability assessment due to its intermittent nature and displacement of a significant portion of the synchronous generation. Moreover, as distribution networks continue in hosting DG units, the electrical distances between generator nodes and load nodes become larger, which can cause major changes in the stability of distribution networks [1]. In addition, some types of DG units, especially fixed speed wind turbines, always consume reactive power, which may cause long-term voltage stability in distribution networks [2]. It is also known that distribution networks continuously witness fast load increase with a slow expansion in its grid, which can also affect voltage stability in distribution networks. Another essential issue is that when transmission networks meet accidents, the voltage stability in distribution systems can significantly be affected during post disturbances periods [3]. For all these reasons, it is expected that future distribution networks will face several challenges in system operation and stability.

Voltage stability margin (VSM) is defined as the minimum distance between the current operating point and the maximum loadability point [4]. The methods of voltage stability analysis that have been developed are mainly oriented to high voltage networks. These methods can be classified into modal analysis techniques and nodal analysis techniques. Comparatively, voltage stability of distribution networks had a less attention by researches. Most of researchers focused on improving voltage profiles for distribution networks. However, a good voltage profile cannot guarantee that the network is stable since voltage magnitude is not a good indicator of voltage stability [5].

Several continuation power flow methods have been developed for voltage stability assessment in radial distribution networks [6],[7]. However, these methods need complex calculations that are not appropriate for online applications. A voltage stability index was introduced for DG optimal placement and sizing in [8]. A voltage stability index for mesh distribution network using circuit analysis theory and biquadratic method is proposed in [9]. Several bus and line indices were introduced for voltage stability assessment in radial distribution networks [10]-[13]. However, most of these indices are based on two-bus equivalent model and none of them takes into consideration the interactions among load buses.

Impedance match technique is widely used in voltage stability monitoring of transmission networks [14],[15]. These techniques are based on measuring the local voltage and current phasors at a network bus, and then the equivalent parameters of the rest of the network seen from this bus is derived. However, these techniques are not able to include the dynamic nonlinear nature of loads in multi-bus power system. Moreover, if these methods are used for voltage stability monitoring in future distribution networks, they will not be able to capture the availability/ unavailability of DG units.

To the best of our knowledge, no research paper in the literature used a real time monitoring for voltage stability in distribution networks that can capture the DG unavailability and takes into consideration the dynamic nonlinear nature of loads. To solve this problem, this work uses a nodal model, which is extracted from kirchhoffs current law, for voltage stability monitoring in multi bus power network using wide area measurements and SCADA system. The proposed model can be used to estimate the distance to voltage 
instability in distribution networks and determine the weak buses based on impedance match theorem. Moreover, it can be easily used for online applications that enable the distribution network operators to take appropriate corrective/ preventive actions for voltage instability mitigation, such as reactive power control, rather than waiting for actions taken by the operator of the high voltage network.

This paper is organized as follows: Section II uses the nodal method to calculate the equivalent impedance of the entire distribution network seen by a load bus, and derives an index for voltage stability margin. Voltage stability monitoring procedure is presented in section III. Section IV explains the studied system and shows the simulation results, followed by conclusion.

\section{EQUIVALENT NODAL MODEL}

Based on power flow equations, we can derive an equivalent nodal model for voltage stability monitoring. This method aims to simplify the distribution network seen by a load bus to an equivalent impedance as shown in Fig.1. The calculated impedance can be used to estimate the voltage stability margin in distribution networks. PMU and SCADA data are used to achieve this task.

According to Kirchhoff's current law, the nodal current equation can be expressed as:

$$
[\mathrm{I}]=[\mathrm{Y}][\mathrm{V}]
$$

where $[\mathrm{I}]$ is the vector of the injected bus currents, $[\mathrm{V}]$ is the vector of bus voltages, and [Y] is the admittance matrix of distribution network. The network buses can be classified into load buses L, Tie buses T, and generator buses G. Thus, nodal current equation can be rewritten as:

$$
\left[\begin{array}{c}
I_{G} \\
-I_{L} \\
0
\end{array}\right]=\left[\begin{array}{lll}
Y_{G G}^{\prime} & Y_{G L}^{\prime} & Y_{G T}^{\prime}{ }_{G T}^{\prime} \\
Y_{L G}^{\prime} & Y_{L L}^{\prime} & Y_{L T}^{\prime} \\
Y_{T G}^{\prime} & Y_{T L}^{\prime} & Y_{T T}^{\prime}
\end{array}\right]\left[\begin{array}{c}
V_{G} \\
V_{L} \\
V_{T}
\end{array}\right]
$$

By eliminating the tie buses, the nodal current equation can be expressed by:

$$
\left[\begin{array}{c}
I_{G} \\
-I_{L}
\end{array}\right]=\left[\begin{array}{cc}
Y_{G G} & Y_{G L} \\
Y_{L G} & Y_{L L}
\end{array}\right]\left[\begin{array}{c}
V_{G} \\
V_{L}
\end{array}\right]
$$

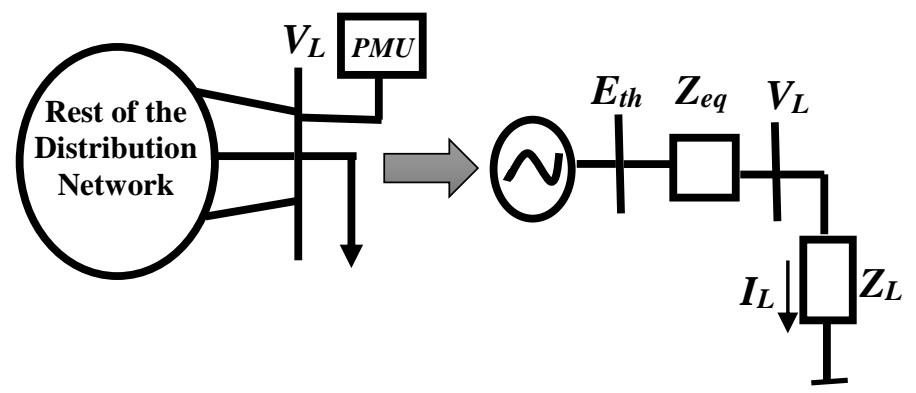

Figure.1 Reduction of distribution network, seen by a load bus.
Where :

$$
\begin{aligned}
& Y_{G G}=Y_{G G}^{{ }^{\prime}}{ }_{G G} Y_{G T}{ }_{G T} Y_{T T}{ }^{-1} Y_{T G}{ }_{T G} \\
& Y_{G L}=Y_{G L}^{\prime}-Y_{G T}^{*} Y_{T T}^{\prime}{ }^{-1} Y_{T L}^{\prime} \\
& Y_{L G}=Y_{L G}^{*}-Y_{L T}^{*} Y_{T T}^{*}{ }^{-1} Y_{T G}^{*} \\
& Y_{L L}=Y_{L L}^{\prime}-Y_{L T}{ }_{L T} Y_{T T}{ }^{-1} Y_{T L}{ }_{T L}
\end{aligned}
$$

Equation (3) can be expressed as:

$$
\left[\begin{array}{c}
I_{G} \\
V_{L}
\end{array}\right]=\left[\begin{array}{cc}
Y_{G G-} Y_{G L} Z_{L L} Y_{L G} & Y_{G L} Z_{L L} \\
-Z_{L L} Y_{L G} & Z_{L L}
\end{array}\right]\left[\begin{array}{c}
V_{G} \\
-I_{L}
\end{array}\right]
$$

Where

$$
\begin{aligned}
Z_{L L} & =Y_{L L}^{-1} \\
& =\left(Y_{L L}^{\star}-Y_{L T}^{{ }^{\prime} Y_{T T}{ }_{T T}^{-1} Y_{T L}}\right)^{-1}
\end{aligned}
$$

From (4), the load voltage can be expressed as

$$
\begin{aligned}
V_{L} & =\left(-Z_{L L} Y_{L G}\right) V_{G}+Z_{L L}\left(-I_{L}\right) \\
& =K V_{G}-Z_{L L} I_{L}
\end{aligned}
$$

Where $K=-Z_{L L} Y_{L G}$. Thus, the voltage at load bus i:

$$
V_{L, i}=\left[K V_{G}\right]_{i}-Z_{L L, i i} I_{L, i}-\sum_{i \neq j} Z_{L L, i j} I_{L, j}
$$

where $I_{L, i}$ and $I_{L, j}$ are the current injected at load buses $\mathrm{i}$ and $\mathrm{j}$ respectively; $Z_{L L, i i}$ is the self-impedance of load buses $\mathrm{i}$; $Z_{L L, i j}$ is the coupling impedance between bus $\mathrm{i}$ and $\mathrm{j}$. Equation (6) can be written as:

$$
V_{L, i}=V_{e q, i}-Z_{e q, i} I_{L, i}
$$

Where

$$
\begin{gathered}
V_{e q, i}=\left[K V_{G}\right]_{i} \\
Z_{e q, i}=Z_{L L, i i}+\sum_{i \neq j} Z_{L L, i j} \frac{I_{L, j}}{I_{L, i}}
\end{gathered}
$$

Where $V_{e q, i}, Z_{e q, i}$ are the Thevenin`s equivalent voltage and impedance seen by the load bus i, respectively. Based on the Thevenin impedance theory, the maximum power transfer to a load bus is obtained when the load impedance equals the equivalent impedance of the rest of the network. i.e. $\left|Z_{\text {eq }}\right|=$ $\left|Z_{L}\right|$. Based on Thevenin's impedance match, a voltage stability index VSI can be formulated as:

$$
\operatorname{VSI}_{i}=\frac{\left|z_{e q, i}\right|}{\left|z_{L, i}\right|}
$$

Where $Z_{L, i}$ is the impedance of bus $i$ and can be calculated as:

$$
Z_{L, i}=\frac{V_{L, i}}{I_{L, i}}
$$


$V_{L, i}$ and $I_{L, i}$ are the measured voltage and current at bus $\mathrm{i}$ respectively. The value of the VSI ranges from 0 to 1 , and $\mathrm{VSI}_{\mathrm{i}}=1$ represents the instability point where equivalent impedance matches the load impedance. The largest value among all the VSIs can be used to determine the system margin and determine the weakest area. Based on that, VSM of the system can be calculated as:

$$
\mathrm{VSM}=1-\max \left(\mathrm{VSI}_{i}\right)
$$

\section{Voltage Stability Monitoring Procedure}

To obtain the equivalent nodal model of a distribution network, the proposed method requires to use PMU at each interested load bus to measure the current and voltage phasors. The network data are collected by a SCADA system. The proposed algorithm is implemented in distribution network control center to enable the operators to take appropriate actions for voltage stability corrective. The procedure for the proposed voltage stability monitoring is as follows:

- Collect the data of admittance matrix of the distribution network and DG unavailability from SCADA system and gather the phasor measurements of current and voltage at the interested buses from PMUs.

- $\quad$ Calculate $Z_{L L}$ using (5).

- Calculate the load impedance using (9) and the equivalent Thevenin impedance using (7.b) for each interested load bus.

- Calculate the voltage stability index for each interested load bus using (8).

- $\quad$ Select the smallest voltage stability margin to be the weak bus in the system using (10).

Fig.2 shows a flowchart for the proposed voltage stability monitoring.

\section{SimUlation RESUlts}

To examine the accuracy of the proposed technique, 77bus, $11 \mathrm{kV}$ distribution network shown in Fig. 3 is taken as a test system in this work. The system consists of 22 DG units and 53 load bus. The line parameters and load data are available in [16]. In this study, all DG units are considered to operate with constant reactive power and do not take part in voltage control. The system and the proposed method have been simulated in MATLAB under different scenarios.

\section{A. Case I: DG Unavailability}

In this scenario, all DG units are considered in the service. Tripping of some DG units is made to show how voltage stability of distribution networks is affected by DG unavailability and how the proposed index can easily reflect the voltage stability margin. Fig. 4 shows a comparison of VSI values for load buses in case of tripping DGs at node 1066, and DGs at 1062 \& 1059.

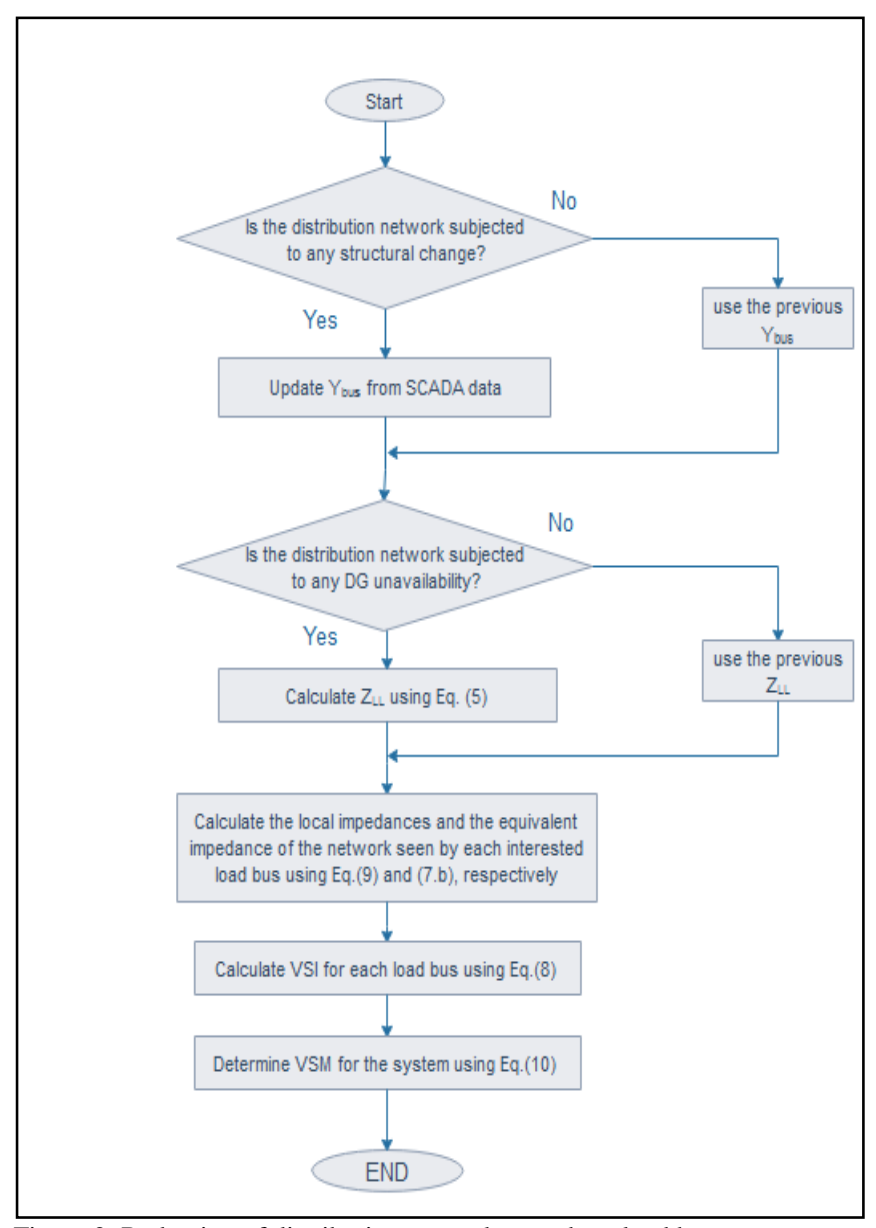

Figure.2 Reduction of distribution network, seen by a load bus.

From Fig. 4, it is clear that the voltage stability margin is changed based on the number and location of DGs tripping. It is also shown that when a DG at a specific bus is tripped, the near-load buses are the most influenced buses. This is an indicator that the proposed method can effectively estimate the voltage stability margin in electric distribution grids during DG unavailability cases.

Regarding the weak buses, it is shown that the smallest voltage stability margin occurs at bus 1113 during normal case, at bus 1176 in cases of DG_1166 tripping, and at bus 1174 in case of DGs_(1059\&1062) tripping. This means that the proposed index can effectively determine the weak buses based on the operational conditions in distribution systems.

If DG tripping is extended to include the three DGs (located at 1059, 1062, \&1066), the estimated VSI values will significantly increase toward voltage instability point as shown in Shown in Fig.5. 


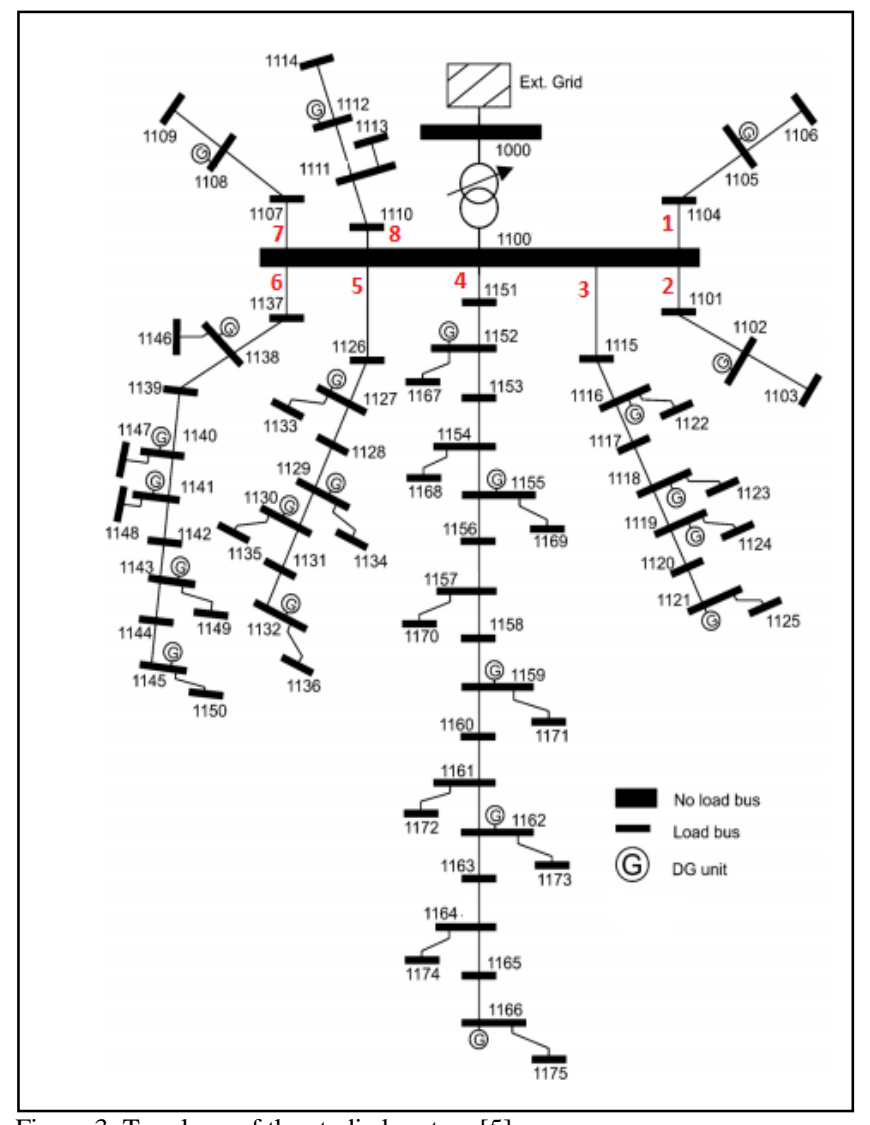

Figure.3 Topology of the studied system [5]

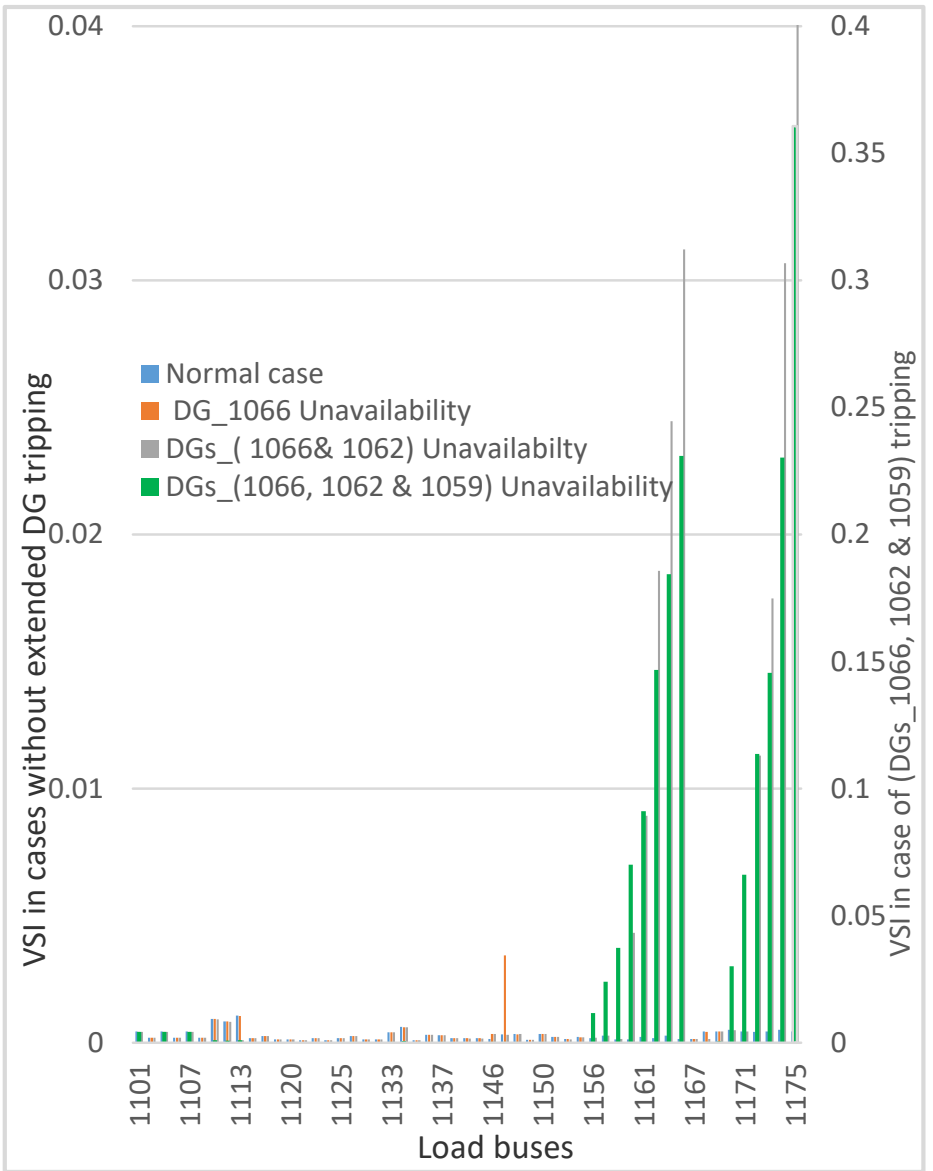

Figure.5 a comparison of estimated VSI during DG tripping cases (including extended DG tripping)

\section{B. Case II: Load increase}

In this scenario, only six DG units are chosen to be in the service (DGs located at 1102, 1108, 1116, 1129, 1155 \& 1062). Fig. 6 compares VSI values of load buses at different percent of load increase $(30 \% \& 60 \%)$. It is clear that the proposed index can accurately estimate the voltage stability margin inside distribution networks. The results show that as load increase, the voltage stability margin decreases. Regarding the weak buses, it is shown that the smallest voltage stability margin occurs at bus 1150 .

\section{Case III: Voltage stability margin Vs voltage profile}

In this scenario, the only six DG units are also chosen to be in the service to prove that voltage magnitude is not a good indicator of voltage stability. Fig.7 compares the estimated values of VSM with voltage profile values for feeder 6- load buses. It is shown that voltages are almost 1.0 p.u and there is overvoltage at some load buses. However, the values of VSM exhibit a significant decrease toward voltage instability. This provides a rigorous justification to the necessity of monitoring voltage stability besides voltage profile in future distribution networks.

It is worth mentioning that corrective measures should be taken by distribution network operators to guarantee that the system is far away from instability point. 


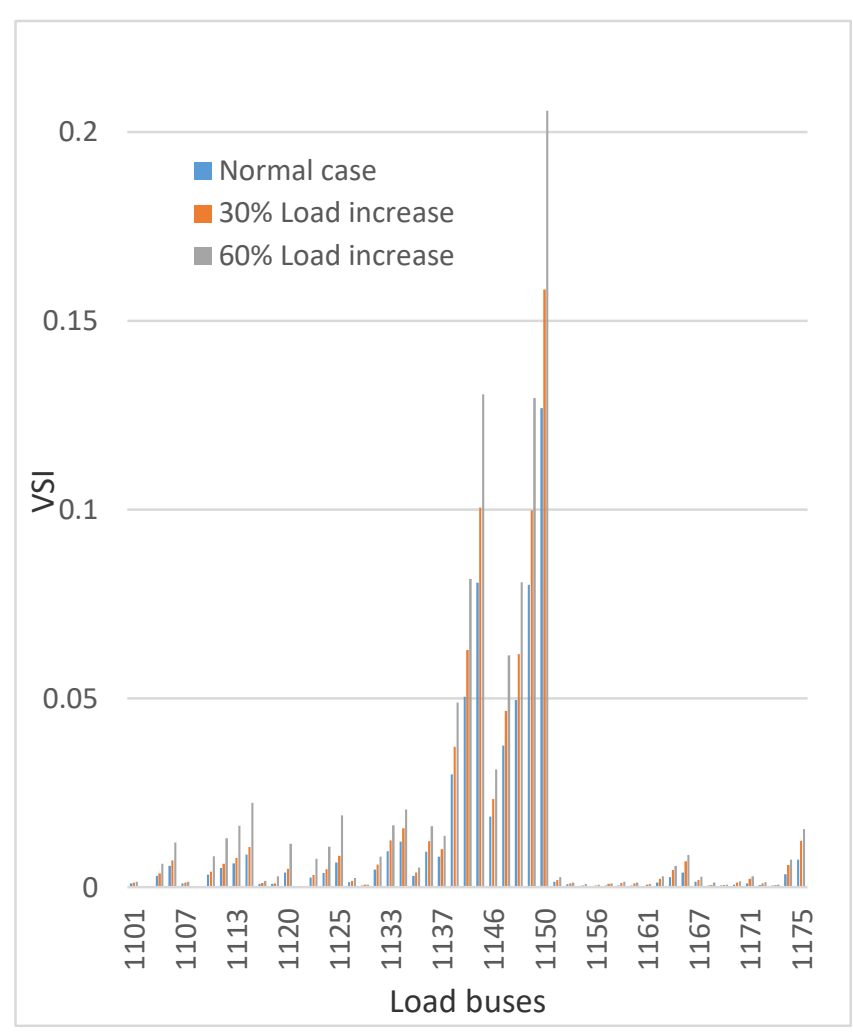

Figure. 6 a comparison of estimated VSI during different percent of load increase.

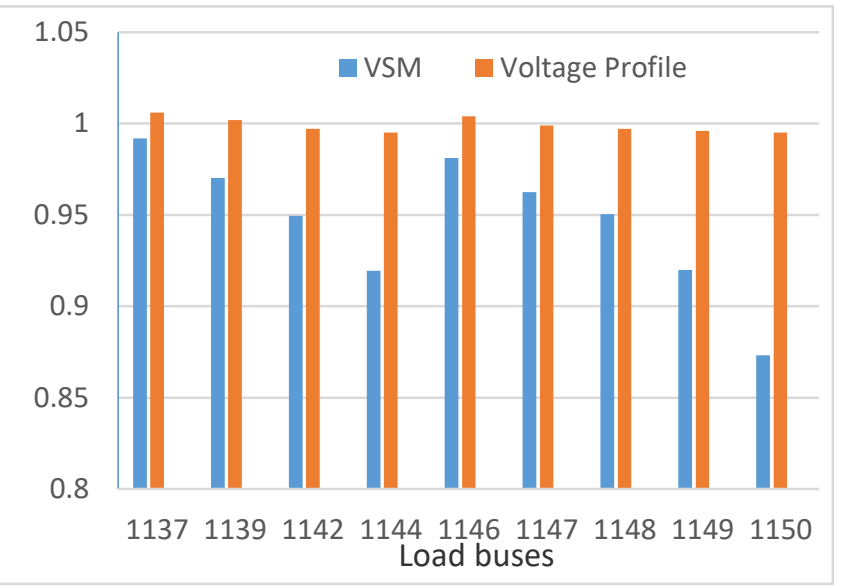

Figure. 7 a comparison of estimated voltage stability margin with voltage profile values for feeder 6- load buses.

\section{CONCLUSION}

As future distribution networks host high penetration levels of distributed generation, the operational characteristics of distribution networks will become similar to transmission networks, and can lead to major changes in the system stability of distribution systems. The proposed method needs only one snapshot of the voltage and current phasors of the interested load buses and SCADA data to estimate the voltage stability margin and determine the weak buses.
The proposed method uses the nodal model to accurately calculate the equivalent impedance seen by a load bus, takes into consideration the nonlinear dynamic nature of the loads and the unavailability of DG units. The proposed technique enables the distribution network operator to take real time corrective actions to mitigate the voltage instability, rather than waiting for actions taken by the operator of the high voltage network.

In the future, our trend is to find a model for real time voltage control in distribution network to improve voltage stability margin.

\section{REFERENCES}

[1] Nazari, Masoud Honarvar, and Marija Ilic, “ Dynamic modelling and control of distribution energy systems: comparison with transmission power systems, " IET Generation, Transmission \& Distribution 8.1, pp.26-34, 2014

[2] Liu, J.H. and Chu, C.C., " Long-term voltage instability detections of multiple fixed-speed induction generators in distribution networks using synchrophasors," IEEE Transactions on Smart Grid, vol. 6, no.4 , pp.2069-2079,2015.

[3] Aristidou, P., Valverde, G. and Van Cutsem, T., "Contribution of distribution network control to voltage stability: A case study," IEEE Transactions on Smart Grid, vol.8, no.1, pp.106-116, 2017

[4] Bahmanyar, A. R., and A. Karami, " Power system voltage stability monitoring using artificial neural networks with a reduced set of inputs, "International Journal of Electrical Power \& Energy Systems 58, pp. 246-256. 2014.

[5] P. A. L"of, T. Smed, G. Andersson, and D. J. Hill, "Fast calculation of a voltage stability index," IEEE Trans. Power Syst., vol. 7, no. 1, pp. 54-64, Feb. 1992.

[6] M. Abdel-Akher, "Voltage stability analysis of unbalanced distribution systems using backward/forward sweep load-flow analysismethod with secant predictor," IET Gen., Transm. Distrib., vol. 7, no. 3, pp. 309-317, 2012.

[7] A. Dukpa, B. Venkatesh, and M. EI-Hawary, "Application of continuation power flow method in radial distribution systems," Electr. Power Syst. Res.ems, vol. 79, pp. 1503-1510, 2009.

[8] V. V. S. N. Murty and A. Kumar, "Optimal placement of DG in radial distribution systems based on new voltage stability index under load growth," Elec. Power Syst. Res., Vol. 69, pp. 246-56, Jul. 2015.

[9] Kazmi, S.A.A., Shahzaad, M.K. and Shin, D.R., "Voltage stability index for Distribution Network connected in Loop Configuration". IETE Journal of Research, 63(2), pp.281-293, 2017.

[10] Gubina, F., Strmcnik, B.: " A simple approach to voltage stability assessment in radial networks, " IEEE Trans. Power Syst., 12, pp. $1121-1128,1997$.

[11] M. Chakravorty, and D. Das, "Voltage stability analysis of radial distribution networks," Int. J. Elec. Power Energy Syst., Vol. 23, pp. 129-35, Feb. 2001.

[12] J. Yu, W. Li, and W. Yan, "A new line loadability index for radial distribution systems," Elec. Power Compo. Syst., Vol. 36, pp. 124552, Oct. 2008.

[13] Chaturvedi, A., Prasad, K., Ranjan, R., "A new voltage stability index for radial distribution network," Int. J. Power Energy Syst., 26, (1), pp. 83- 88, 2006.

[14] K. Vu, M. Begovic, D. Novosel, andM. Saha, "Use of local measurements to estimate voltage-stability margin," IEEE Trans. Power Syst., vol. 14, no. 3, pp. 1029-1035, Aug. 1999.

[15] A. Wiszniewski, "New criteria of voltage stability margin for the purpose of load shedding," IEEE Trans. Power Del., vol. 22, no. 3, pp. 1367-1371, Jul. 2007.

[16] United Kingdom Generic Distribution Network (UKGDS). [Online]. Available: http://sedg.ac.uk 